\title{
ASdeCopas: a syntactic-semantic interface ${ }^{\star}$
}

\author{
Luísa Coheur $^{1,2}$, Nuno Mamede ${ }^{1}$, and Gabriel G. Bès ${ }^{2}$ \\ ${ }^{1}$ L $^{2}$ F INESC-ID/IST - Spoken Languages Systems Laboratory \\ \{luisa. coheur $\{$ numo.mamede $\}$ l2f . inesc-id.pt \\ 2 GRIL/Université Blaise-Pascal \\ Gabriel.Bes@univ-bpclermont.fr
}

\begin{abstract}
ASdeCopas is a syntactic-semantic parser, implemented in Prolog, which uses hierarchically organized order-independent rules. This paper focuses on the formalization of semantic rules, presenting the concepts of well-formed semantic rule, rules hierarchy, and the conditions for rules application. If two rules can apply, only the most specific one does so. Examples are given and some properties of the system are pointed out.
\end{abstract}

Keywords: syntactic-semantic interface, semantic rules, rules hierarchy, 5P, Minimal Recursion Semantics

\section{Introduction}

ASdeCopas $^{3}$ is a syntactic-semantic parser that takes a graph representing the input sentence and returns a formula, according with a set of semantic rules. The paper focuses on the formalization of semantic rules. Section 2 describes ASdeCopas's input, semantic rules are formalized in section 3, section 4 presents simplified examples and section 5 lists some of the system properties and discusses perspectives on future work.

\section{ASdeCopas's Input}

Ideas, formalisms and data from the $5 \mathrm{P}$ paradigm $[1,3,6,2]$ are followed/used to obtain ASdeCopas' input: a text with an associated graph. A graph is defined as follows (let $C$ be a set of category labels, $W$ a set of words and $\_$the empty field):

Definition 1. Graph

A graph is a pair $G=(\Delta, \Psi)$, where:

- $\Delta$ is a set of nodes, each one noted node $(w, c, p)$, where $w \in W, c \in C$ and $p$

$\in \mathbb{N}$ (p represents the node's position).

- $\Psi$ is a set of arrows, each arrow noted arrow $\left(p_{1}, p_{2}\right)$, where $p_{1}, p_{2} \in \mathbb{N}\left(p_{1}, p_{2}\right.$ being the position of the source and the target, respectively).

\footnotetext{
* Paper supported by FCT (Fundação para a Ciência e Tecnologia).

3 ASdeCopas stands for "Análise Semântica depois de Completada a análise sintáctica".
} 
A well formed graph (wfg) verifies:

- $\left(\forall \operatorname{arrow}\left(p_{1}, p_{2}\right) \in \Psi\right)\left(\exists \operatorname{node}\left(w_{1}, c_{2}, p s_{1}\right), \operatorname{node}\left(w_{2}, c_{2}, p s_{2}\right) \in \Delta\right)\left(p_{1}=p s_{1}\right.$ $\left.\wedge p_{2}=p s_{2}\right)$ (that is, each arrow connects existing nodes)

- $\left(\forall \operatorname{arrow}\left(p_{1}, p_{2}\right), \operatorname{arrow}\left(p_{3}, p_{4}\right) \in \Psi\right)\left(p_{1}=p_{3} \Rightarrow p_{2}=p_{4}\right)($ that is, each node is the source of at most one arrow)

- $\forall \operatorname{node}\left(w_{1}, c_{1}, p_{1}\right), \operatorname{node}\left(w_{2}, c_{2}, p_{2}\right), \operatorname{node}\left(w_{3}, c_{3}, p_{3}\right), \operatorname{node}\left(w_{4}, c_{4}, p_{4}\right) \in$ $\Delta)\left[\left(p_{1}<p_{2}<p_{3}<p_{4} \wedge\left(\exists \operatorname{arrow}\left(p_{1}, p_{3}\right) \vee \exists \operatorname{arrow}\left(p_{3}, p_{1}\right) \in \Psi\right)\right) \Rightarrow \neg(\exists\right.$ $\left.\left.\operatorname{arrow}\left(p_{2}, p_{4}\right) \vee \exists \operatorname{arrow}\left(p_{4}, p_{2}\right) \in \Psi\right)\right]($ that is, no crossing of arrows is allowed)

Each category is a set of attribute/value pairs ${ }^{4}$, i.e., feature structures hierarchically organized (see [1] for details). Notice that no constraint is set on the nature of those pairs: they can have a syntactic or a semantic motivation. Arrows are somehow related to dependencies, but, contrary to mainstream dependency theories, arrows go from dependents to the head [6]. Their motivation is simply to connect two elements, because the established relations are needed to reach the desired semantic representation (see $[1,3]$ for extra details about this concept). An example of a graph is shown in the next figure, where $A$ pequena Maria means Little Mary:
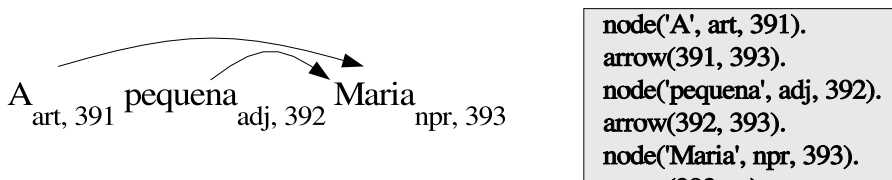

Fig. 1. Graph example.

\section{Semantic rules}

Definitions 2 to 8 define the syntax of a well-formed semantic rule, while Definitions 9 to 11 define rules hierarchy and Definitions 12 and 13 rules applicability conditions.

Definition 2. Element (to transform)

An element has the form $e=\operatorname{elem}(w, c)$, where $w \in\{-\} \cup W$ and $c \in\{-\} \cup C$.

Definition 3. Arc

An arc has the form $a=\operatorname{arc}\left(c_{1}, c_{2}, d\right)$, where:

- $c_{1}, c_{2} \in C\left(c_{1}\right.$ and $c_{2}$ are, respectively, the source and the target of the arc);

- $d \in\{-\} \cup\{L, R\}$ ( $L$ when the arc goes from right to left, $R$ from left to right).

${ }^{4}$ For expository reasons we use a unique label to identify those sets. 
Definition 4. Semantic Rule

A semantic rule is a triple $R_{i}=(\Sigma, \Theta, \Gamma)$ (also notated $\left[R_{i}\right] \Sigma: \Theta \leftrightarrow \Gamma$ ), where:

- $\Sigma$ is a (non empty) set of elements;

- $\Theta$ is a (possibly empty) set of arcs;

- $\Gamma$ is a set of translating functions. ${ }^{5}$

For the following definitions, let $R_{i}=(\Sigma, \Theta, \Gamma)$ be a semantic rule.

Definition 5. Connections between elements Let $e_{1}=\operatorname{elem}\left(w_{1}, c_{1}\right), e_{2}=\operatorname{elem}\left(w_{2}, c_{2}\right) \in \Sigma$.

$e_{1}$ and $e_{2}$ are said to be directly connected $\left(e_{1} \bowtie_{e} e_{2}\right)$ if and only if (iff) there is an arc $a=\operatorname{arc}\left(c_{3}, c_{4}, d\right) \in \Theta$, such that

- $\left(c_{1}=c_{3} \wedge c_{2}=c_{4}\right) \vee\left(c_{1}=c_{4} \wedge c_{2}=c_{3}\right)$

$e_{1}$ and $e_{2}$ are said to be connected $\left(e_{1} \bowtie_{e}^{*} e_{2}\right)$ iff

- $\left(e_{1} \bowtie_{e} e_{2}\right) \vee\left(\exists e_{3} \in \Sigma\right)\left(e_{1} \bowtie_{e} e_{3} \wedge e_{3} \bowtie_{e}^{*} e_{2}\right)$

Definition 6. Connections between arcs

Let $a_{1}=\operatorname{arc}\left(c_{1}, c_{2}, d_{1}\right), a_{2}=\operatorname{arc}\left(c_{3}, c_{4}, d_{2}\right) \in \Theta$ and $a_{1} \neq a_{2}$.

$a_{1}$ and $a_{2}$ are said to be directly connected $\left(a_{1} \bowtie_{a} a_{2}\right)$ iff

- $c_{1}=c_{4} \vee c_{2}=c_{3} \vee c_{2}=c_{4}$

$a_{1}$ and $a_{2}$ are said to be connected $\left(a_{1} \bowtie_{a}^{*} a_{2}\right)$ iff

- $\left(a_{1} \bowtie_{s} a_{2}\right) \vee\left(\exists a_{3} \in \Theta\right)\left(a_{1} \bowtie_{a} a_{3} \wedge a_{3} \bowtie_{a}^{*} a_{2}\right)$

Definition 7. Relation arc/element

Let $a_{1}=\operatorname{arc}\left(c_{1}, c_{2}, d_{1}\right) \in \Theta$ and $e=\operatorname{elem}(w, c) \in \Sigma$.

$a_{1}$ and $e$ are said to be directly connected $\left(a_{1} \rightarrow e\right)$ iff

- $c=c_{1} \vee c=c_{2}$

$a_{1}$ and $e$ are said to be connected $\left(a_{1} \rightarrow^{*}\right.$ e) iff

- $\left(a_{1} \rightarrow e\right) \vee\left(\exists a_{2} \in \Theta\right)\left(a_{1} \bowtie_{a}^{*} a_{2} \wedge a_{2} \rightarrow e\right)$

Definition 8. Well formed semantic rule

$R_{i}=(\Sigma, \Theta, \Gamma)$ is a well formed semantic rule (wfsr) iff:

- $\left(\forall e_{1}, e_{2} \in \Sigma\right)\left(e_{1} \bowtie_{e}^{*} e_{2}\right)$ (all the elements are connected)

- $\left(\forall a_{1} \in \Theta\right)(\exists e \in \Sigma)\left(a_{1} \rightarrow^{*} e\right)$ (all the arcs are related with an element)

Example 1. Well formed semantic rule

The following rule, whose target is Minimal Recursion Semantics (MRS) [5], is a wfsr ( $\mathrm{n}$ is the label of the category associated with nouns):

$$
\left[R_{1}\right]\left\{\operatorname{elem}\left(\_, \mathrm{n},{ }_{-}\right)\right\}: \emptyset \rightarrow\{\operatorname{handle}(\mathrm{n}): \operatorname{sem}(\mathrm{n})(\operatorname{var}(\mathrm{n}))\}
$$

For the following definitions let $\sqsubseteq$ be the subsumption relation between two sets.

Definition 9. Element subsumption

$e_{1}=\operatorname{elem}\left(w_{1}, c_{1}\right)$ subsumes $e_{2}=\operatorname{elem}\left(w_{2}, c_{2}\right)\left(e_{1} \sqsubseteq e e_{2}\right)$ iff $\left(c_{1} \sqsubseteq c_{2}\right) \wedge\left(w_{1} \neq \neq_{-} \Rightarrow w_{2}=w_{1}\right)$

\footnotetext{
${ }^{5}$ For expository reasons we will not detail translating functions. However, consider defined $\operatorname{sem}(\mathrm{c}), \operatorname{var}(\mathrm{c})$ and handle(c), returning, respectively, the semantics, a variable and an handle [5] associated with the element identified by category c.
} 
Definition 10. Arc subsumption

$a_{1}=\operatorname{arc}\left(c_{1}, c_{2}, d_{1}\right)$ subsumes $a_{2}=\operatorname{arc}\left(c_{3}, c_{4}, d_{2}\right)\left(a_{1} \sqsubseteq a a_{2}\right)$ iff

$\left(c_{1} \sqsubseteq c_{3} \wedge c_{2} \sqsubseteq c_{4}\right) \wedge\left(d_{1} \neq{ }_{-} \Rightarrow d_{1}=d_{2}\right)$

Definition 11. Rule subsumption

Let $R_{1}=\left(\Sigma_{1}, \Theta_{1}, \Gamma_{1}\right), R_{2}=\left(\Sigma_{2}, \Theta_{2}, \Gamma_{2}\right)$ be wfsr.

$R_{1}$ subsumes $R_{2}\left(R_{1} \sqsubseteq_{r} R_{2}\right)$ iff

$\left(\forall e_{1} \in \Sigma_{1}\right)\left(\exists e_{2} \in \Sigma_{2}\right)\left(e_{1} \sqsubseteq_{e} e_{2}\right) \wedge\left(\forall a_{1} \in \Theta_{1}\right)\left(\exists a_{2} \in \Theta_{2}\right)\left(a_{1} \sqsubseteq_{a} a_{2}\right)$

Example 2. Rule subsumption

As $\mathrm{n} \sqsubseteq \mathrm{np}$, rule $R_{1}$ subsumes the following rule:

$\left[R_{2}\right]\left\{\operatorname{elem}\left({ }_{-}, \mathrm{np},{ }_{-}\right)\right\}: \emptyset \rightarrow\{$ handle $(\mathrm{np}): \operatorname{NAME}(\operatorname{var}(\mathrm{np}), \operatorname{sem}(\mathrm{np}))\}$

Definition 12. Conditions for the application of a semantic rule

Let $R_{j}=(\Sigma, \Theta, \Gamma)$ be a wfsr and $G=(\Delta, \Psi)$ a wfg. $R_{j}$ can apply to $G$ iff:

- $\left(\forall \operatorname{elem}\left(w_{i}, c_{i}\right) \in \Sigma\right)\left(\exists \operatorname{node}\left(w_{j}, c_{j}, p_{j}\right) \in \Delta\right)\left[\left(w_{i} \neq \ldots\right.\right.$

- $\left(\forall \operatorname{arc}\left(c_{n}, c_{m}, d\right) \in \Theta\right)\left(\exists \operatorname{arrow}\left(p_{k}, p_{l}\right) \in \Psi, \exists \operatorname{node}\left(w_{k}, c_{k}, p_{k}\right), \operatorname{node}\left(w_{l}, c_{l}\right.\right.$,

$\left.\left.p_{l}\right) \in \Delta\right)\left(c_{n} \sqsubseteq c_{k} \wedge c_{m} \sqsubseteq c_{l} \wedge\left(d=R \Rightarrow p_{k}<p_{l}\right) \wedge\left(d=L \Rightarrow p_{k}>p_{l}\right)\right)$

Definition 13. Application of a semantic rule

Being given a wfg, let $R_{1}$ and $R_{2}$ be wfsr, verifying the conditions to be applied to it. If $R_{1} \sqsubseteq_{r} R_{2}$, then $R_{1}$ is not applied.

Example 3. Semantic rules applicability

Both rules $R_{1}$ and $R_{2}$ are in conditions to be applied to the graph from Fig. 1.

As $R_{1} \sqsubseteq_{r} R_{2}$ only $\mathrm{R}_{2}$ is triggered.

\section{Example}

If rule $R_{1}$ is applied to the graph from Fig. 1, the following formula is obtained: $\mathrm{h}_{393}$ : Maria $\left(\mathrm{x}_{393}\right)$ (notice that variable generation is not carried out randomly: variable indexes are given by the position of the associated element or of the element it arrows). However, as only $R_{2}$ is triggered, we obtain: $\mathrm{h}_{393}$ : NAME ( $\mathrm{x}_{393}$, Maria).

Next we present a rule for intersective and subsective adjectives [4] (we will use the notation from [7]). ${ }^{6}$

$\left[R_{3}\right]\left\{\operatorname{elem}\left({ }_{-}, \operatorname{adj},-\right.\right.$ ) $\}:\{\operatorname{arc}(\operatorname{adj}, \mathrm{n})\} \rightarrow\{\operatorname{handle}(\mathrm{n}): \operatorname{AM}(\operatorname{var}(\mathrm{n}), \operatorname{sem}(\operatorname{adj}))\}$ After adding this rule to the system, the following formula is generated is addition to the previous: $\mathrm{h}_{393}: \operatorname{AM}\left(\mathrm{x}_{393}\right.$, pequena $)$.

\section{Brief discussion and future work}

Briefly, ASdeCopas has the following properties:

\footnotetext{
${ }^{6}$ We should point out that reification of variables [8] over adjectives will be needed to allow modification. However, we will ignore this problem for expository reasons.
} 
- propagation of ambiguous values can be precluded through the use of (syntactic) information presented in the rules;

- rules can be applied in any order, as they are intrinsically independent (that is, their output does not depend on the output of other rule);

- information can be modularly added by profiting from the hierarchical organization of both categories and semantic rules;

- partial results can be produced;

- different semantic processes (such as role extraction, anaphora resolution, ...) can run at different times and their final results merged, due to the controlled generation of variables. ${ }^{7}$

The main problem with this system is that the immediate production of a structured formula is not easy, even though equivalent "flatter" structures can be easily produced. Moreover, the production of partial results should be taken carefully, as inconsistent representations can be produced.

ASdeCopas is implemented in Prolog and is being tested in question interpretation and in a more formal framework where the output is Minimal Recursion Semantics.

\section{References}

1. Gabriel G. Bès. La phrase verbal noyau en français. In Recherches sur le français parlé, 15, pages 273-358. Université de Provence, France, 1999.

2. Gabriel G. Bès. Empiricité en linguistique et grammaire de montague: la sémantique en $5 \mathrm{P}$ et la compositionnalité (avec en annexe grammaire de montague et ambiguïté). Technical report, GRIL, Université Blaise-Pascal, Clermont-Ferrand, France, Avril, 2001.

3. Gabriel G. Bès and Caroline Hagège. Properties in 5P (soon in the GRIL web page). Technical report, GRIL, Clermont-Ferrand, France, November, 2001.

4. Gennaro Chierchia and Sally McConnell-Ginet. Meaning and Grammar - an Introduction to Semantics (second edition). The MIT Press, 2000.

5. Ann Copestake, Dan Flickinger, and Ivan A. Sag. Minimal Recursion Semantics. An introduction. CSLI, Stanford University. 1997.

6. Caroline Hagège. Analyse Syntatic Automatique du Portugais. PhD thesis, Université Blaise Pascal, Clermont-Ferrand, France, 2000.

7. Daniel Jurafsky and James Martin. Speech and Language Processing. Prentice Hall, 2000.

8. Diego Mollá. Ontologically promiscuous flat logical forms for NLP. In IWCS-4, Tilburg, The Netherlands, 2000.

\footnotetext{
${ }^{7}$ We are not assuming DRT to solve anaphora.
} 